\title{
Topological system method of formation of transformer transformation coefficients
}

\author{
Dauren S. Akhmetbayev ${ }^{1, *}$, Abdigali R. Dzhandigulov ${ }^{2}$, and Svetlana V. Bystrova ${ }^{3}$ \\ ${ }^{1}$ Kazakh Agrotechnical University, Electric Power Supply Department, Nur-Sultan, Kazakhstan \\ ${ }^{2}$ Eurasian National University, Algebra and Geometry Department, Nur-Sultan, Kazakhstan \\ ${ }^{3}$ Ekibastuz Engineering and Technical Institute named after Ak. K. Satpayev, Ekibastuz, Republic of Kazakhstan
}

\begin{abstract}
A systematic approach to determining the control range of the on-load tap-changer of transformers from the standpoint of the synthesis of the voltage mode is proposed. Transformations of the equation of nodal voltages, formalized on the basis of the topology of a complex electrical network, with respect to the transformation ratios of transformers are given.
\end{abstract}

Key words. topology, nodal equation, current distribution coefficients, transformation ratio of transformers.

\section{Introduction}

An increase in the efficiency of power consumers can be achieved at normalized values of the power quality indicators $[1,2]$. In order to ensure the standard indicators of the quality of electricity, continuous regulation of the network voltage mode is required, which is associated with great difficulties due to the variety of regime tasks. The choice of the most expedient methods of regulating the voltage regime depends on local conditions, and is made based on a technical and economic comparison of possible options for the application of certain methods or means. The analysis of the study shows that the greatest economic effect is obtained from centralized voltage regulation at power points of distribution networks [3, 4, and 5].

One of the main methods of voltage regulation in electrical networks is the use of regulating devices at stepdown substations, operating on the principle of counter regulation. Power transformers with a voltage of $35 \mathrm{kV}$ and above are equipped with a special device for voltage regulation - a regulator under load (OLTC), which is an automatic device that changes the working branch of the transformer winding turns. The adjustable winding can have 12.16 or 18 steps of regulation by 1.5 or $1.78 \%$ with a regulation range of $\pm 9, \pm 12$ or $\pm 16 \%$ of $U_{\text {nom }}[5,6]$.

To increase the efficiency of using the on-load tapchanger of transformers, it is necessary to check the sufficiency of the regulating range to ensure the desired voltage level on the low-voltage bus bars in the modes of the highest and lowest loads. This problem is solved individually for each transformer based on the results of calculating the steady state of electrical networks of power systems $[7,8]$.

Notwithstanding the above-specified ranges of adjustment of working branches achieve desired voltage level using tap changer with a deficit of reactive power is impossible. Therefore, the development of a systemic method for assessing the adjustment range of the on-load tap-changer of transformers is an urgent task in modern conditions of voltage control of electrical networks.

\section{Topological equations of node voltages}

The solution to this problem can be obtained by transforming the topological system of nodal stresses [9]. Based on the theory of graphs, a method for the formation of generalized parameters of the electric network, in particular, the matrix of the distribution coefficients of the driving currents is proposed [10]. The developed topological algorithms are implemented in the form of a software package for searching and determining all possible and specific trees of a directed graph of a complex electrical network $[11,12]$. The matrix of the distribution coefficients of the driving currents is formalized based on the topological expression $[10,11$ and 12]:

$$
\underline{C}_{i j}=\frac{\sum F_{i j}}{\sum F}
$$

where $\sum F_{i j}$ - the algebraic sum of specific i-branch trees relative to the $\mathrm{j}$-th node;

$\sum F$ - an arithmetic sum of all possible graph trees.

Node voltages are determined by topological expression obtained by analytical transformation of known equations of electric state of networks [13-16]:

\footnotetext{
*Corresponding author: axmetbaev46@mail.ru
} 


$$
\dot{U}=\dot{U}_{0}-\underline{C}^{t} \underline{Z}_{\partial} \underline{C}_{\partial} \hat{U}^{-1} \hat{S}
$$

where $\underline{C}$ - matrix of complex coefficients of assignment currents distribution; $\underline{Z}_{\partial}$ - matrix of complex coefficients of assignment currents distribution; $\dot{U}_{0}$ - base node voltage; $\hat{U}_{\partial}$ - diagonal matrix of a conjugated complex of node voltages; $\hat{S}$ - vector of a conjugated complex of nodal loads; $t$ - sign of matrix transposition.

\section{Modelling transformation ratios of transformers}

Suppose that the regulating ability of the on-load tapchanger of the transformers provides the desired voltage on the bus bars of the secondary winding of the transformer in the modes of the highest and lowest loads. Therefore, the value of the desired voltage on the bus bars of the step-down substation can be considered a given value. This allows us to formulate the problem of modelling the transformation ratios of transformers from the standpoint of the synthesis of permissible modes for the voltage of electrical networks of the power system. Formalization of the problem posed can be achieved by transforming the topological equation of nodal stresses (2).

Suppose that the desired voltages on the buses of transformer substations, switched on for the first time by $\mathrm{n}$ nodes of a complex electrical network, are provided by changes in the transformation ratios of transformers. Then the nonlinear matrix equation (2), after simple transformations, will be written in the form of a voltage balance, for example, for the i-th node [17]:

$$
\begin{aligned}
& \dot{U}_{\text {ides }}=-\dot{U}_{\Delta i 1} K_{i} K_{1}-\dot{U}_{\Delta i 2} K_{i} K_{2}-\cdots-\dot{U}_{\Delta i i} K^{2}{ }_{i}-\cdots \\
& +\left(\dot{U}_{0}-\sum_{k=n+1}^{N} \dot{U}_{\Delta i k}\right) K_{i}
\end{aligned}
$$

where

$\dot{U}_{\Delta i 1}=\sum_{j=1}^{m} \underline{C}_{i j}^{t} \underline{Z}_{j} \underline{C}_{j 1} \hat{U}_{1 \operatorname{des}}^{-1} \hat{S}_{1}, \dot{U}_{\Delta i n}=\sum_{j=1}^{m} \underline{C}_{i j}^{t} \underline{Z}_{j} \underline{C}_{j n} \hat{U}_{n d e s}^{-1} \hat{S}_{n}$,

$\dot{U}_{\Delta i k}=\sum_{j=1}^{m} \underline{C}_{i j}^{t} \underline{Z}_{j} \underline{C}_{j k} \hat{U}_{k}^{-1} \hat{S}_{k}$ - partial nodal voltage drops of the $i$-th node;

$\dot{U}_{\text {ides }}$ - complex desired voltage of the $i$-th node;

$U_{0}$ - voltage of the basic node;

$K_{1}, K_{2}, \ldots, K_{n}$ - transformation ratios of transformers;

$N$ - the number of independent nodes.

The dependent variables in equation (3) are the phases of the nodal voltages $(\delta)$ and the transformation ratios of the transformers $(K)$.

The system of nonlinear equations for the stress balance of the node under consideration with respect to the variables $(\delta, K)$ can be obtained from (3), highlighting the real and imaginary parts, in the form $[14,15]$ :

$$
\left.\begin{array}{l}
\omega_{U_{i}^{\prime}}(\delta, K)=U_{i d e s} \cos \delta_{i}+\sum_{j=1}^{n} U_{\Delta i j} K_{i} K_{j} \cos \left(\delta_{j}-\varphi_{j}+\psi_{i j}\right)- \\
-\left(U_{0}-\sum_{k=n+1}^{N} U_{\Delta i k} \cos \left(\delta_{k}-\varphi_{k}+\psi_{i k}\right)\right) K_{i} \\
\omega_{\dot{U}_{i}^{\prime \prime}}(\delta, K)=U_{i d e s} \sin \delta_{i}+\sum_{j=1}^{n} U_{\Delta i j} K_{i} K_{j} \sin \left(\delta_{j}-\varphi_{j}+\psi_{i j}\right)+ \\
+\left(\sum_{k=n+1}^{N} U_{\Delta i k} \sin \left(\delta_{k}-\varphi_{k}+\psi_{i k}\right)\right) K_{i}
\end{array}\right\}
$$

where $U_{i}^{\prime}, U_{i}^{\prime \prime}$ are the real and imaginary parts of the voltage;

$U_{\Delta i j}$ - module of partial nodal voltage drop;

$\varphi_{j}$ - phase shift of the load power of the $\mathrm{j}$-th node;

$\psi_{i j}$ - angle of mutual complex nodal resistance.

It can be seen from (4) that a system of $2 n$ equations with $2 \mathrm{n}$ unknowns is compiled, the solution of which can be obtained by Newton's method. The Jacobi matrix is not degenerate and can be written in block form as:

$$
\frac{\partial \mathbf{W}_{U}(x)}{\partial x}=\| \begin{array}{ll}
\frac{\partial \omega_{v^{\prime}}(\delta, K)}{\partial \delta} & \frac{\partial \omega_{v^{\prime}}(\delta, K)}{\partial K} \\
\frac{\partial \omega_{v^{\prime \prime}}(\delta, K)}{\partial \delta} & \frac{\partial \omega_{v^{\prime \prime}}(\delta, K)}{\partial K} \|
\end{array}
$$

If we neglect for distribution networks the influence of the phases of the nodal voltages on the values of the driving currents, then system (4) can be written as:

$$
\left.\begin{array}{l}
\omega_{\underline{\dot{U}}_{i}^{\prime}}(\delta, K)=U_{i d e s} \cos \delta_{i}+\sum_{j=1}^{n} U_{\Delta i j} K_{i} K_{j} \cos \left(-\varphi_{j}+\psi_{i j}\right)+ \\
-\left(U_{0}-\sum_{j=n+1}^{N} U_{\Delta i j} \cos \left(-\varphi_{j}+\psi_{i j}\right)\right) K_{i} \\
\omega_{\underline{\dot{U}}_{i}^{\prime \prime}}(\delta, K)=-U_{i d e s} \sin \delta_{i}+\sum_{j=1}^{n} U_{\Delta i j} K_{i} K_{j} \sin \left(-\varphi_{j}+\psi_{i j}\right)+ \\
+\left(\sum_{j=n+1}^{N} U_{\Delta i j} \sin \left(-\varphi_{j}+\psi_{i j}\right) K_{i}\right.
\end{array}\right\}
$$

Then the partial derivatives of the matrix are defined as:

$$
\begin{aligned}
& \frac{\partial \omega_{v^{\prime} i}(\delta, K)}{\partial \delta}=U_{i d e s} \sin \delta_{i} ; \\
& \frac{\partial \omega_{v^{\prime} i}(\delta, K)}{\partial K}=2 U_{\Delta i i} K_{i} \cos \left(-\varphi_{i}+\psi_{i i}\right)+ \\
& +\sum_{j=n+1}^{N} U_{\Delta i j} \cos \left(-\varphi_{j}+\psi_{i j}\right)-U_{0}+ \\
& +\sum_{\substack{j=1 \\
j \neq i}}^{n} U_{\Delta i j} K_{i} \cos \left(-\varphi_{j}+\psi_{i j}\right) ;
\end{aligned}
$$




$$
\begin{aligned}
& \frac{\partial \omega_{v^{\prime \prime} i}(\delta, K)}{\partial \delta}=-U_{i d e s} \cos \delta_{i} ; \\
& \frac{\partial \omega_{v^{\prime \prime} i}(\delta, K)}{\partial K}=2 U_{\Delta i i} K_{i} \sin \left(-\varphi_{i}+\psi_{i i}\right)+ \\
& +\sum_{j=n+1}^{N} U_{\Delta i j} \sin \left(-\varphi_{j}+\psi_{i j}\right)+ \\
& +\sum_{\substack{j=1 \\
j \neq i}}^{n} U_{\Delta i j} K_{i} \sin \left(-\varphi_{j}+\psi_{i j}\right) .
\end{aligned}
$$

When calculating distribution networks, the formulas for determining the elements of the Jacobi matrix are significantly simplified and reduced in the amount of calculations performed, since the equation for the voltage of the $\mathrm{i}$-th node can be reduced to the form:

$$
\begin{aligned}
& \omega_{U_{i}}(K)=U_{i \varkappa c}+\sum_{j=1}^{n} U_{\Delta i j} K_{i} K_{j} \cos \left(-\varphi_{j}+\psi_{i j}\right)- \\
& \left(U_{0}-\sum_{k=n+1}^{N} U_{\Delta i k} \cos \left(-\varphi_{j}+\psi_{i k}\right)\right) K_{i}
\end{aligned}
$$

Then the partial derivatives of the matrix are defined as:

$$
\begin{aligned}
& \frac{\partial \omega_{v^{\prime} i}(K)}{\partial K}=2 U_{\Delta i i} K_{i} \cos \left(-\varphi_{i}+\psi_{i i}\right)+ \\
& +\sum_{j=n+1}^{N} U_{\Delta i j} \cos \left(-\varphi_{j}+\psi_{i j}\right)-U_{0}+ \\
& +\sum_{j=1}^{n} U_{\Delta i j} K_{i} \cos \left(-\varphi_{j}+\psi_{i j}\right) ;
\end{aligned}
$$

The system of equations for the zero iteration is written as follows:

$$
W\left(K^{0}\right)+\frac{\partial W\left(K^{0}\right)}{\partial K} \Delta K^{(1)}=0
$$

where $\Delta K^{(1)}$ is the matrix of corrections of the first Newton iteration. Each step of the iterative process involves solving system (9) with the subsequent determination of the approximation:

$$
K^{(i+1)}=K^{i}+\Delta K^{(i+1)}
$$

In general, Newton's iterative process can be written as:

$$
K^{(i+1)}=K^{(i)}-\left[\frac{\partial W\left(K^{i}\right)}{\partial K}\right]^{-1} W\left(K^{i}\right)
$$

Convergence control is carried out using the vector of residuals:

$$
W_{v}(K) \leq \varepsilon,
$$

where $\varepsilon$ is a predetermined small value.

\section{Determination of the stage of the control branch}

According to the calculated values of the transformation ratios, it is easy to find the standard step number of the transformer control tap according to the formula [8]:

$$
n=\frac{\frac{1}{K} \cdot U_{R N}-U_{H N}}{U_{A d d} \% \cdot U_{R N}} \cdot 100
$$

Where

$U_{H N}, U_{R N}$ - rated voltage of high and low voltage transformer winding;

$$
U_{\text {Add }} \text { - voltage addition of one step. }
$$

The standard number of the control step is determined by rounding to the nearest whole number. For the found branch number, the actual value of the desired voltage of the secondary winding of the transformer is determined.

The sufficiency of the control capacity of the transformer is assessed with a given range of on-load tapchangers. If the on-load tap-changer adjustment range is insufficient, it is necessary to evaluate the value of the capacitor bank power required on the low voltage buses.

\section{Algorithm research results}

As an example, two substations are considered, powered from a $110 \mathrm{kV}$ ring network. At substation 1 there are two transformers with a capacity of 25 MVA each, and at substation 2 - with a capacity of 16 MVA each. Transformers $110 \mathrm{kV}$ with on-load tap-changer have a switching device for voltage regulation with nine steps of $1.78 \%$. It is required to determine the transformation ratios and regulating taps of the on-load tap-changer of transformers for the given load modes, indicated in Table 1 The design diagram of the network with parameters is shown in Fig. 1a. The directed network graph is shown in Fig. 1b.

The developed complex program is used to search and determine the weights of all possible and specific trees in the graph [18]. According to the graph trees, a matrix of distribution coefficients of the driving currents is determined, the values of which are equal [19]:

$$
C=\left(\begin{array}{cccc}
1 & 0 & 0 & 0 \\
0 & 1 & 0 & 0 \\
-0.5747+0.0122 i & -0.3138-0.0409 i & -0.5747+0.0122 i & -0.3138-0.0409 i \\
-0.4253-0.0122 i & 0.3138+0.0409 i & -0.4253-0.0122 i & 0.3138+0.0409 i \\
-0.4253-0.0122 i & -0.6862-0.0409 i & -0.4253-0.0122 i & -0.6862-0.0409 i
\end{array}\right)
$$

Then a nonlinear system of equations for the transformation ratios of transformers (7) is formalized, which is solved on the basis of known methods, for example, Newton's method. Comparative results of calculations performed for the scheme in Fig. 1 with parameters are presented in Table 1. 

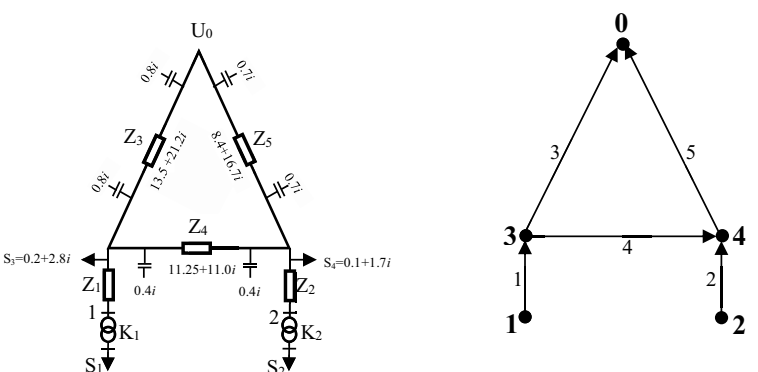

Fig. 1a) Design diagram of a network with parameters b) directed graph

Table 1. Calculation results of transformation ratios and control taps

\begin{tabular}{|c|c|c|c|c|c|c|c|c|}
\hline \multirow{2}{*}{$№$} & \multicolumn{2}{|c|}{$\begin{array}{c}\text { Node } \\
\text { voltages }\end{array}$} & \multicolumn{4}{c|}{ Transformation ratios } \\
\cline { 2 - 9 } & $\mathrm{S}_{1}$ & $\mathrm{~S}_{2}$ & $\mathrm{U}_{0}$ & $\mathrm{U}_{*}$ & \multicolumn{2}{|c|}{$\mathrm{k} 1$} & \multicolumn{2}{|c|}{ k2 } \\
\cline { 2 - 9 } & $\mathrm{MB} \times \mathrm{A}$ & $\mathrm{MB} \times \mathrm{A}$ & $\mathrm{kB}$ & $\mathrm{kB}$ & $\mathrm{CM}$ & $\mathrm{SM}$ & $\mathrm{CM}$ & $\mathrm{SM}$ \\
\hline 1 & $42+21 \mathrm{i}$ & $28+7 \mathrm{i}$ & 125 & 10,5 & 0,093 & 0,093 & 0,092 & 0,090 \\
\hline 2 & $36+18 \mathrm{i}$ & $24+6 \mathrm{i}$ & 125 & 10,5 & 0,092 & 0,091 & 0,089 & 0,089 \\
\hline 3 & $30+15 \mathrm{i}$ & $20+5 \mathrm{i}$ & 125 & 10,5 & 0,091 & 0,090 & 0,088 & 0,088 \\
\hline 4 & $24+12 \mathrm{i}$ & $16+4 \mathrm{i}$ & 115 & 10 & 0,093 & 0,093 & 0,091 & 0,092 \\
\hline 5 & $18+9 \mathrm{i}$ & $12+3 \mathrm{i}$ & 115 & 10 & 0,091 & 0,091 & 0,090 & 0,091 \\
\hline 6 & $12+6 \mathrm{i}$ & $8+2 \mathrm{i}$ & 115 & 10 & 0,090 & 0,090 & 0,089 & 0,089 \\
\hline
\end{tabular}

Table 1. Continuation
\begin{tabular}{|c|c|c|c|c|c|c|c|c|}
\hline \multirow{2}{*}{$№$} & $\begin{array}{c}\text { Substation } \\
\text { loads }\end{array}$ & \multicolumn{2}{c|}{$\begin{array}{c}\text { Node } \\
\text { voltages }\end{array}$} & \multicolumn{3}{c|}{ Regulating branch number } \\
\cline { 2 - 9 } & $\mathrm{S}_{1}$ & $\mathrm{~S}_{2}$ & $\mathrm{U}_{0}$ & $\mathrm{U}_{*}$ & \multicolumn{2}{|c|}{$\mathrm{n} 1$} & \multicolumn{2}{c|}{$\mathrm{n} 2$} \\
\cline { 2 - 9 } & $\mathrm{MB} \cdot \mathrm{A}$ & $\mathrm{MB} \cdot \mathrm{A}$ & $\mathrm{kB}$ & $\mathrm{kB}$ & $\mathrm{CM}$ & $\mathrm{SM}$ & $\mathrm{CM}$ & $\mathrm{SM}$ \\
\hline 1 & $42+21 \mathrm{i}$ & $28+7 \mathrm{i}$ & 125 & 10,5 & 1,4 & 1,84 & 3,43 & 3,85 \\
\hline 2 & $36+18 \mathrm{i}$ & $24+6 \mathrm{i}$ & 125 & 10,5 & 2,45 & 2,76 & 4,15 & 4,45 \\
\hline 3 & $30+15 \mathrm{i}$ & $20+5 \mathrm{i}$ & 125 & 10,5 & 3,45 & 3,65 & 4,83 & 5,04 \\
\hline 4 & $24+12 \mathrm{i}$ & $16+4 \mathrm{i}$ & 115 & 10 & 1,67 & 1,84 & 2,43 & 2,27 \\
\hline 5 & $18+9 \mathrm{i}$ & $12+3 \mathrm{i}$ & 115 & 10 & 2,73 & 2,82 & 3,65 & 3,14 \\
\hline 6 & $12+6 \mathrm{i}$ & $8+2 \mathrm{i}$ & 115 & 10 & 3,73 & 3,78 & 4,33 & 3,98 \\
\hline
\end{tabular}

\section{Conclusions}

The study showed that

1) The developed and implemented algorithms allow determining the transformation ratios of transformers with high accuracy.

2) The developed program can be used for design and operational calculations of the modes of electrical networks.

\section{References}

1. Venikov V.A., Idelchik V.I., Liseev M.S. Voltage regulation in electric power systems. M .: Energoaotmizdat, 1985.-120s.

2. Regulation of voltage in electrical networks. Reports of N.A. Melnikov, N.S. Markushevich, Ya.D. Barkon, L.A. Soldatkina. M .: Energy, 1968.-606s.

3. Soldatkina L.A. Voltage regulation in urban networks. M .: Energy, 1972.-272s.

4. Arkhipov N.K. Voltage mode in electrical distribution networks. M .: publishing house VZEI, 1964.-131s.

5. Zhelezko Yu.S. Determination of power and places of local voltage control installation//Electrical stations. Moscow. 1972.- No. 11.- C.34-36.

6. Barkan Ya.D. Voltage regulation automation in distribution networks. M .: Energy, 1972.-120s.

7. Zhelezko Yu.S. Electricity losses. Reactive power. Power quality. M .: Publishing house NTs ENAS, 2009.420 s.

8. Lykin A.V. Electrical systems and networks. Novosibirsk: Publishing house of NSTU, 2017.-363s
9. Geraskin O.T. Graphs of an electrical network and topological formulas // Izvestiya AN SSSR. Energy and Transport. 1971.- No. 4.- P.66-75.

10. D.S. Akhmetbayev, D.0A. Aubakir, Y.Zh. Sarsikeyev, B.A. Bainiyazov, M.A. Surkov, V.I. Rozhko, G.N. Ansabekova, A.S. Yerbolova, A.T. Suleimenov, M.S. Tokasheva. Development of topological method for calculating current distribution coefficients in complex power networks, // Results in Physics, 2017, 7, pp. 16441649.

11. A.R. Dzhandigulov, D.S. Akhmetbaev. Implementation of a new topological algorithm for calculating current distribution coefficients in complex electrical networks. Computer program. Certificate of entering information into the state register of rights to objects protected by copyright. No. 1552 dated January 31, 2019

12. D.S. Akhmetbaev, A.R. Dzhandigulov. Finding all spanning graphs of a given graph. Computer program. Certificate of entering information into the state register of rights to objects protected by copyright. No. 1551 dated January 31, 2019

13. D. Akhmetbayev, A. Akhmetbayev, A. Suleymenov, M. Kolcun. Modeling the set mode of complex power grid, based on infeed coefficients // Proceedings of the 9th International Scientific Symposium «Elektroenergetika2017», 12.-14. 9.2017, Stará Lesná, Slovak Republic.

14. D.S. Akhmetbaev, A.R. Dzhandigulov, A.D. Akhmetbaev. Topological algorithm for forming nodal stresses of complex networks energy systems. Web of Conferences 139, 01066 (2019)

15. D.S. Akhmetbaev; A.D. Akhmetbaev; A.R. Dzhandigulov. New Approachesto the Topological Method of Analysis of Electrical Networks. International Multi-Conference on Industrial Engineering and Modern Technologies, FarEastCon, 2019.

16. D.S. Akhmetbaev, A.R. Dzhandigulov, A.D. Akhmetbaev. Topological algorithm forforming nodal stresses ofcomplex networks energy systems. E3S Web of Conferences 139, 01066 (2019).

17. D. Akhmetbayev, A. Akhmetbayev, A. Aidarova Determination of rational transformation coefficients transformers distribution networks // E3S Web of conferences 25.04003 (2018)

18. D.S. Akhmetbaev, A.R. Dzhandigulov. Development of algorithms for a new topological method for calculating current distribution coefficients in complex electrical networks // Eurasian journal of mathematical and computer applications. ISSN 2306-6172, Volume 7, Issue 3 (2019) 4-12

19. D.S. Akhmetbaev, A.R. Dzhandigulov, A.D. Akhmetbaev. Development of algorithms for the formation of steady-state modes based on the topology of electric power systems // Journal of Physics: Conference Series 1392 (1), 012079, 2019. 